\title{
Multi Trans-Receive Signals with Quad Co-ordinates Performance Scheme
}

\author{
Kuldeep Shukla \\ Electronics \& Communication \\ SAL-ITER \\ Ahmedabad-380060
}

\author{
Nidhi Bhatt \\ Electronics \& Communication \\ SAL-ITER \\ Ahmedabad-380060
}

\author{
Yash Sharma \\ Electronics \& Communication \\ SAL-ITER \\ Ahmedabad-380060
}

\begin{abstract}
In the field of communication, for any transmission and reception of information-signal, very basic element is Antenna. It is a basic need to transmit the information as well as receive the same with multiple hops in the environment. There are several technologies like SISO, MISO, SIMO and MIMO which achieve one or more than one path. If path is more than one for same information then it will produce high efficiency and increase SNR (Signal to Noise Ratio) and reduce BER (Bit Error Rate). In which MIMO is efficient technology to reduce the overall time and also improves the efficiency by taking multiple input and output at the side of broadcast and response. But the major issue is if all the frequencies are occupied and transmit vital information then controller has to find some different way to transmit the information. OFDM removes that disadvantage by taking different phase and divide whole band-width into some narrow subcarrier and then transmit the valuable data into the air.

In this paper we focus how BER decreases and SNR increases with high data rate where transmission and reception having more than one hop and frequencies are orthogonal with each other for saving the overall band-width. So MIMO-OFDM having some light in this paper named Multi Input Multi Output-Orthogonal Frequency Division Multiplexing.
\end{abstract}

\section{Keywords}

Bit Error Rate, Signal to Noise Rate, OFDM, MIMO

\section{INTRODUCTION}

MIMO is the technique in which bandwidth is divided into several narrow frequencies with specific time intervals (guard band). The advantage of this is, it takes less time to transmit as well as receive the same due to its parallel transmission.

Whereas in case of the other technologies i.e. SISO, MISO and SIMO, they face numerous downsides, SISO (Serial transmission and Serial reception) has the limitation of taking more time for overall performance. MISO that stands for Parallel transmission and Serial reception, takes medium time for overall performance and SIMO is for Serial transmission and Parallel reception take medium time for overall performance. Hence these all are more time compared to that of MIMO [1].

The advantage of MIMO is it reduces the overall time and also improves the efficiency by taking multiple input and output at the side of broadcast and response. In this research, concept of MIMO (Multi Input Multi Output) is used. 4G also used this pattern due to accessibility of less bandwidth and high efficiency.

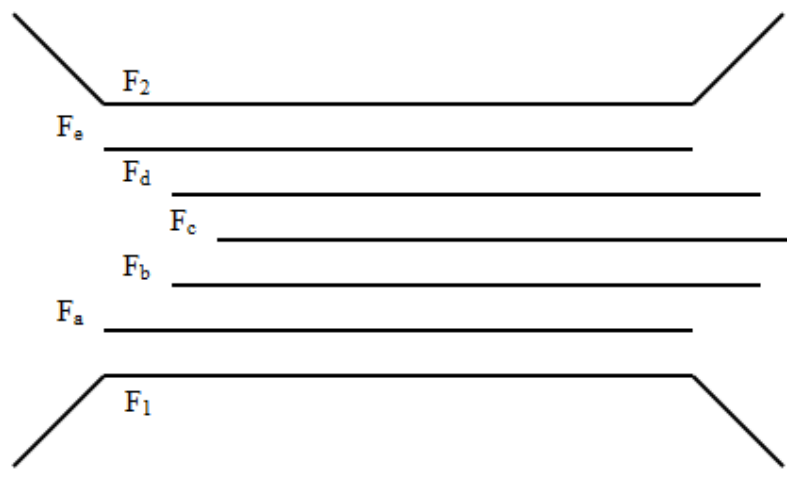

Fig.1 MIMO (Transmission)

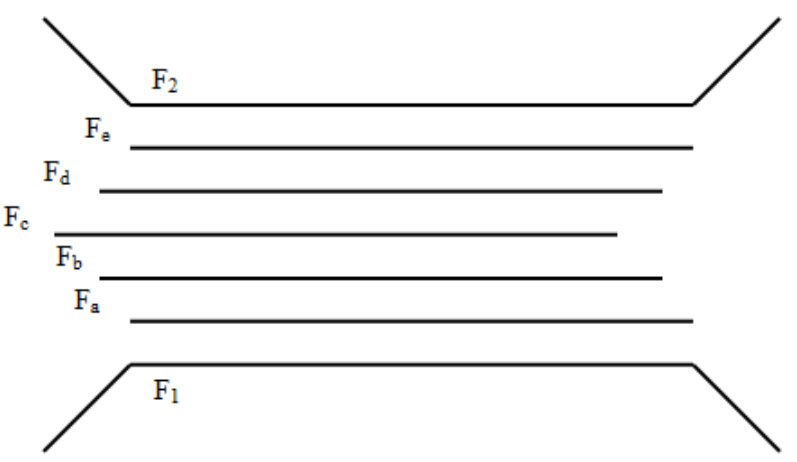

Fig.2 MIMO (Receiver)

The mathematical representation of MIMO Band-Width (B.W.) can be given as the following equation:

$\mathrm{BW}=\mathrm{F}_{2}-\mathrm{F}_{1} ; \mathrm{i}=\mathrm{a}, \mathrm{b}, \mathrm{c}, \mathrm{d}$. .

$$
\text { MIMO B. W. }=\sum_{F_{1}}^{F_{2}} F_{i}
$$

In OFDM system, all the signals are to be orthogonal $\left(90^{\circ}\right)$ to each other. OFDM is identified as "The dot product of two different periodic frequencies in which integration over zero to $T_{b}$ is equal to zero." And signal must be in only one of its kind and identical time space. $T_{b}$ is used to send out the bits of the signal into the air or wireless state of affairs. Because of the zero guard band utilized in orthogonal system, the frequency shifts its position and therefore, covers lesser area than that of in TDM, FDM etc [6].

Mathematical representation of OFDM is:

$$
\int_{0}^{T_{b}} \cos \left(2 \pi f_{1} t\right) \cdot \sin \left(2 \pi f_{2} t\right) d t=>\delta=\frac{2 k}{T_{b}}
$$

Where $\delta=$ difference between two frequency $=\mathrm{f}_{1}-\mathrm{f}_{2}$ 
$\mathrm{k}$ represents the periodic continuity for the given function with all its sub functions and $\mathrm{T}_{\mathrm{b}}$ is defined as a time taken by the length of transmitting bit.[3]

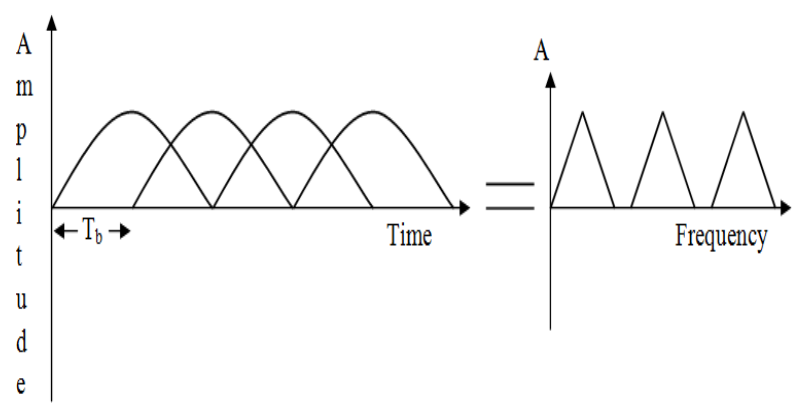

Fig. 3 Time and Frequency domain representation of OFDM output

\section{ISSUES}

In MIMO-OFDM basic four issues crop up as following:

1. All the modulation techniques provide the output in Time domain i.e. the output of QPSK (Quadrature Phase Shift Keying) or QAM (Quadrature Amplitude Modulation) is always in form of signal (time domain).

But in OFDM, at the transmission end the IFFT (Inverse Fast Furrier Transform) is transmitting side so how it is possible to transmit the information because input of IFFT is under in frequency domain. [7], [8]. Consider the figure 4

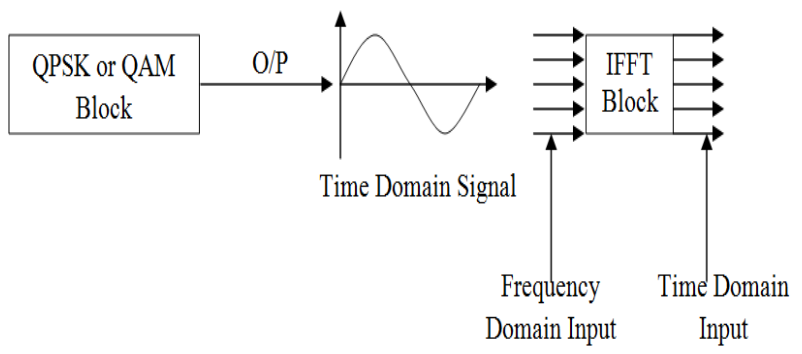

Fig. 4 Issue one: Time Domain--Frequency Domain

2. Harmonics are always present in any signaling system. In OFDM harmonics affect the information signal and produce lake of information or distortion due to overlapping [2], as in following diagram.

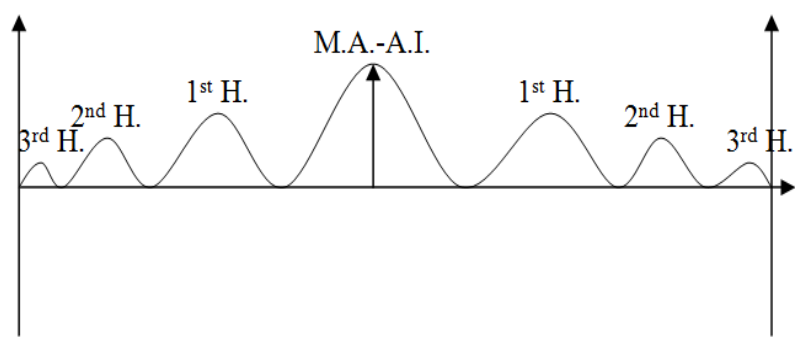

Fig. 5 Issue two: Harmonics Representation

3. The system is wireless, so it may possible that the transmitting system not produce orthogonal (Always 90 degree with each other) signal always due to the effect that two different frequencies overlap to each other for long distance transmission and produce co-channel interference [9].
Noise will easily affect in that case and produce highly indistinct signal at output side. See the figure 6. Occasionally, receiver cannot predict what the signal is and diminish overall efficiency.

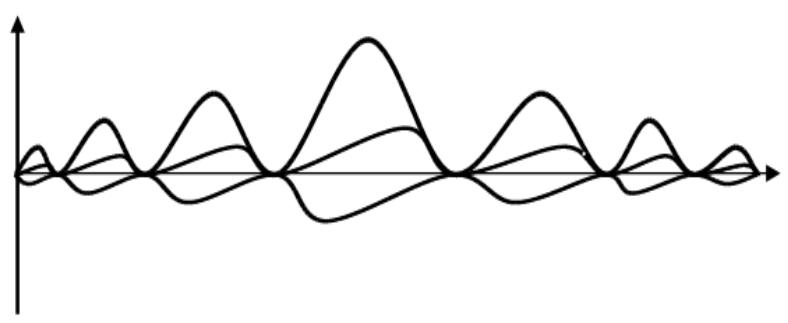

Fig. 6 Issue three: Always 90 degree Phase Shift rarely possible in wireless system

4. When parallel data transmission arises, it may overlap with each other in wireless system as per shown in the below diagram (fig.7).

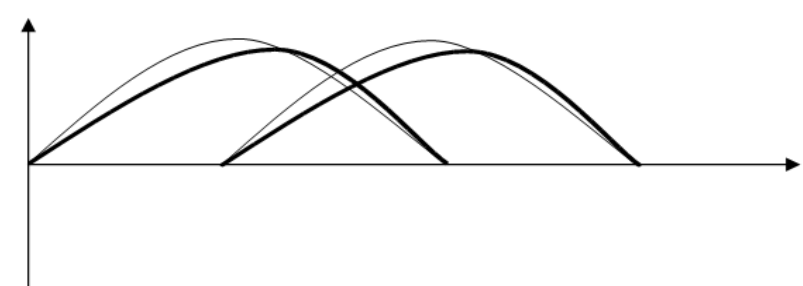

Fig. 7 Issue four: Information overlap and signal distortion

\section{ELUCIDATION}

The so far discussed issues are having some proposals of remedies to reduce the corresponding effects. They include:

1. The output of QPSK or QAM is for eternity represented in time domain but the vector representation of the given signal is in addition possible because of finding magnitude and phase of the instantaneous value for the given episodic signal. The vectored form of any time domain signal is known as frequency for instantaneous value. Horde of that vector representation is called domain of frequency [10].

Vector representation of the signal in frequency domain is:
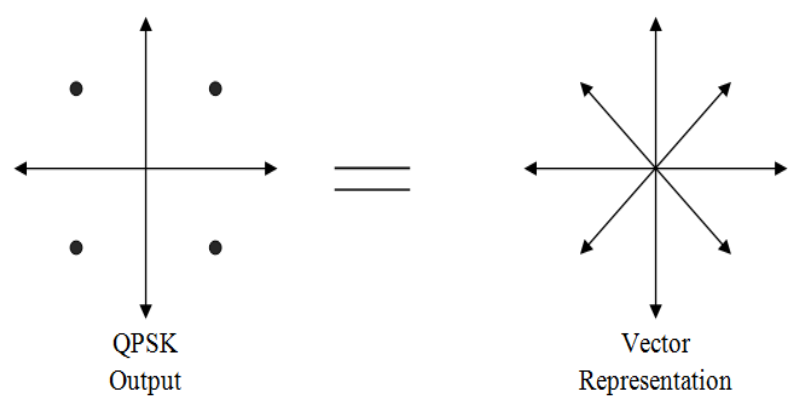

Fig. 8 Output of QPSK

2. In a trouble-free fixation, harmonics are nothing but fading information due to unwanted signal addition in the wireless system. If any function has different time with different frequency and same periodic time or over one time period represents less amplitude with difference $\pm n \pi$. Where $n$ indicates the no. of harmonics and maximum peak has the value $\frac{2 T_{1}}{T_{0}}$. Indication of $T_{0}$ is nothing but a starting time of Fourier signal. 
In mathematical circumstances it is represented by-

$f(t)=a_{0}+\sum_{n=1}^{\infty}\left(a_{n} \cos n \omega_{0} \mathrm{t}+b_{n} \sin \mathrm{n} \omega_{0} \mathrm{t}\right)$

Where, $\left[t_{0} \leq \mathrm{t} \leq t_{0}+\mathrm{T}\right]$

$\mathrm{f}(\mathrm{t})=a_{0}+a_{1} \cos \left(\omega_{0} \mathrm{t}\right)+a_{2} \cos \left(\omega_{0} \mathrm{t}\right)+\cdots+$

$a_{n} \cos \left(\omega_{0} \mathrm{t}\right)+b_{1} \sin \left(\omega_{0} \mathrm{t}\right)+b_{2} \sin \left(\omega_{0} \mathrm{t}\right)+\cdots+b_{k} \sin \left(\omega_{0} \mathrm{t}\right)$

Where, $\mathrm{a}_{0}=$ D.C. Term $=\frac{1}{T} \int_{t_{0}}^{t_{0}+T} f(t) d t$,

$$
\begin{aligned}
& a_{n}=\frac{2}{T} \int_{t_{0}}^{t_{0}+T} f(t) \cos \left(n \omega_{0} t\right) d t \\
& b_{n}=\frac{2}{T} \int_{t_{0}}^{t_{0}+T} f(t) \sin \left(n \omega_{0} t\right) d t
\end{aligned}
$$

If " $n$ " high it means the no. of harmonics are more in that function end if sharp band pass filter is used in that series then it can easily reduce the harmonics it cuts the signal after the value of $n$ is one [11].

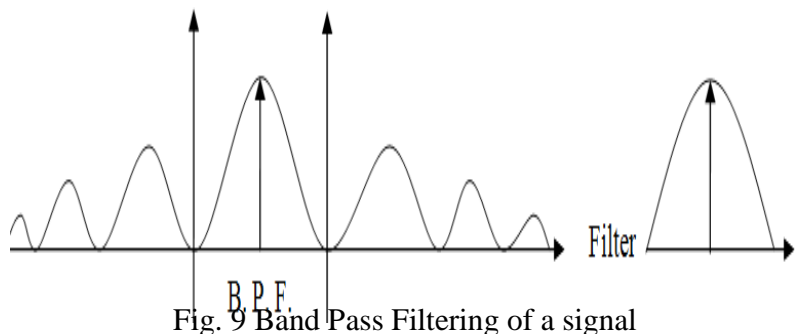

Equation for filter process is:

$$
f(t)=\sum_{k=-\infty}^{+\infty} a_{k} e^{j k \omega_{0} t}
$$

Where $a_{k}=\frac{1}{T_{0}} \int_{0}^{T_{0}} f(t) e^{-j k \omega_{0} t}$

$a_{0}=\frac{2 T_{1}}{T_{0}}, a_{0}=\frac{\sin \left(k \omega_{0} T_{0}\right)}{k \pi} \forall \mathrm{k}$ All sine function for any $\mathrm{k}$

$b_{0}=\frac{\cos \left(k \omega_{0} T_{0}\right)}{k \pi} \forall \mathrm{k}$ all cosine function for any $\mathrm{k}$

3. The minimum separation angle for OFDM is 90 degree. When any signal broadcast into the air then it may possible that it change its phase and overlap with the other Fourier signal. If one bit or more than one bit send out use parity generator and checker to overcome this problem. If more bits have some error in the information signal take more bit parity checker or CRC (Cyclic Redundancy Check) to check the correct output and gives feedback to the transmitter to resend it.

4. To conquer word interference between two words than use trails and footprint or guard-bits. Because of that it protects the words as well as efficient security.

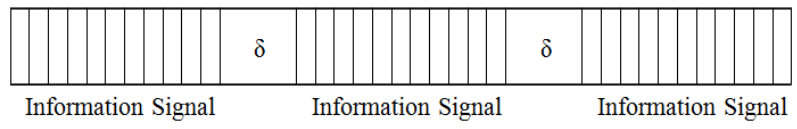

Fig. 10 Frame of OFDM

\section{RESULT ANALYSIS}

According to this simulation, in MATLAB it produce multipath signal with orthogonal frequencies. Those frequencies are considered as an orthogonal with the $L$ Hospital formula of mathematics. Due to this equation, it filters the second maximum harmonic with band pass filter. But here second and third harmonics are also included. The M.A.-A.I. has maximum information in the frequency domain so efficiency is elevated than the previous graph and gives exclusive result in BER v/s SNR [5]. Consider the following simulation result as in figure 11 .

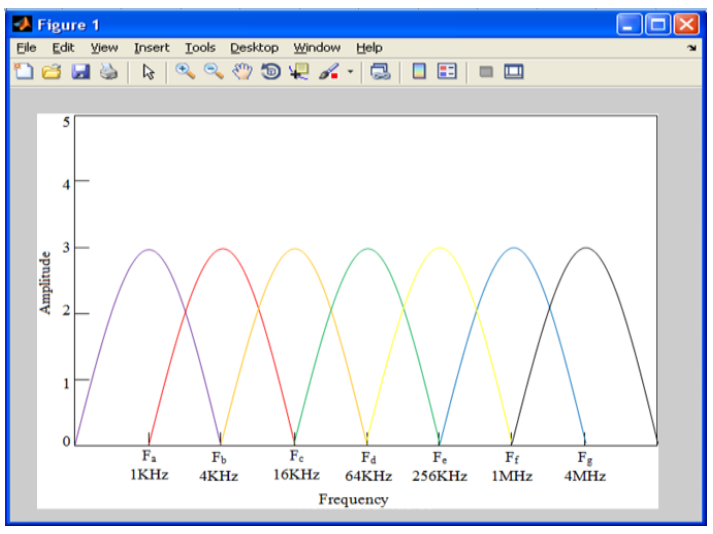

Fig. 11 OFDM generation

Resultant graph of Bit Error Rate (BER) v/s Signal to Noise Ratio (SNR) represents 10 powers few $\mathrm{mm}$ unit in $40 \mathrm{~dB}$ signaling. The output of this process produces higher efficiency over noisy channel. This output also indicates that the information takes always low and secure path in multifunctional parameters.

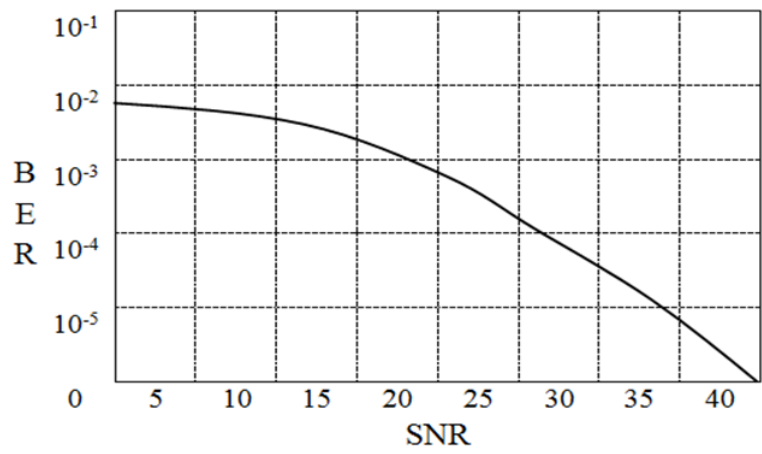

Fig. 12 Resultant graph

If any change in the parameters occurs then it changes its value and reaches to its lowest value in BER to higher value SNR [4].

\section{APPLICATIONS}

The application areas of this technology include the following:

- Due to the so far discussed structure of MIMO OFDM, it is the best applicable in military applications.

- The technologies of Wi-Max and Wi-Fi also accept the process of OFDM due to convenience of obtaining large area cover for single performance of a signal. 
- $4 \mathrm{G}$, a newer generation of cellular phone system is also having application of MIMO-OFDM in signaling process which was limitation in case of $3 \mathrm{G}$ technology.

\section{CONCLUSION}

The discussion carried out through the paper, leads to conclude that the technique of multipath OFDM (i.e. MIMO OFDM) defines lower BER giving high SNR value. It provides lesser system complexity over all kinds of noises in the process of communication. Also, the sanctuary layer elevation becomes easier exclusive of requiring significant changes in performance parameters.

\section{IMPLEMENTATION STRATEGY}

The implementation of the MIMO OFDM can be done in a number of ways. V-BLAST technique is one of them to run MIMO OFDM in efficient way. As a part of future work of this paper we are going to analyze this technique that faces several issues at the time of implementation. The remedies of the issues of MIMO OFDM discussed in this article, do make favors for handling the corresponding issues of V-BLAST technique.

\section{ACKNOWLEDGEMENT}

For the completion of this initial work, the notions of MIMO OFDM, KBS acknowledges to the guides and research scholars for their valuable assistance. We are also thankful to the friends who helped us directly or indirectly.

\section{REFERENCE}

[1] P. Wolniansky, G. Foschini, G. Golden and R. Valenzuela, "V-BLAST, An Architecture for Realizing Very High Data Rates Over The Reach-Scattering Wireless Channel", In Proc. IEEE ISSE, pp.295-300, Sep.1998.

[2] L. Z. Ding, P. Xiaoming and C. Francois, "V-BLAST, Receivers for downlink Mc-CDMA Systems", in proc. IEEE VTC. Fall, pp. 866-870, Sep. 2003.
[3] S. Hemrungrote, T. Hori, M. Fujimoto and K. Nishimori, "Channel Capacity Characteristics of Multi User MIMO System in urban area", 2010-IEEE, 2010

[4] C. H. Binsheng, P. Cheng, Zhu, Xiaohu You, "Energy Efficient Comparisons between Distributed MIMO and co-located MIMO in the uplink cellular systems", 2012IEEE, 2012.

[5] A. Phasouliotis and Daniel K.C.So, "Performance Analysis and Comparison of Downlink MIMO MCCDMA and MIMO OFDMA System", 2009-IEEE, pp.16, 2009

[6] J. M. Chung, J. Kim and D. H. Han, "Multihop Hybrid Virtual MIMO Scheme for Wireless Sensor N/W", 2012IEEE, Vol. 61, Issue-9, Nov. 2012.

[7] Weiyan Ge, J. Zhang and G. Xue, "MIMO pipe modeling and Scheduling for Efficient Interference Management Inter-hop MIMO N/W", IEEE-Oct-2010.Vol.59, Issue-8, 2010.

[8] H. Kusumoto, H. Okada, K. Kobayashi and M. Katayama, "Performance Comparison between Single User MIMO and Multi User MIMO in Wireless Mesh N/W”, IEEE-2011 pp.202-206, 2011.

[9] A. Goldsmith, S. A. Jafar, N. Zindal and S. Vishwanath, "Capacity Limits of MIMO Channels", 2003-IEEE, 2003

[10] E. M. Mohamed, D. Kinoshita, K. Mitsunaga, Y. Higa and H. Furukawa, "MIMO Based Wireless Backhaul", ICUMT- 2010, pp. 202-209, 2010.

[11] H. Yan, GuoweiShen, R. Zetik, O. Hirsch and R. S. Thoma, "Ultra Wideband MIMO Ambiguity Function and Its Factorability", 2012-IEEE.Vol.51, issue-1, pp. 504-519, Jan-2013. 